Document downloaded from:

http://hdl.handle.net/10251/49241

This paper must be cited as:

Alberola Oltra, JM.; Julian Inglada, VJ.; García-Fornes, A. (2014). An intelligent selfconfigurable mechanism for distributed energy storage systems. Cybernetics and Systems. 45(3):292-305. doi:10.1080/01969722.2014.894859.

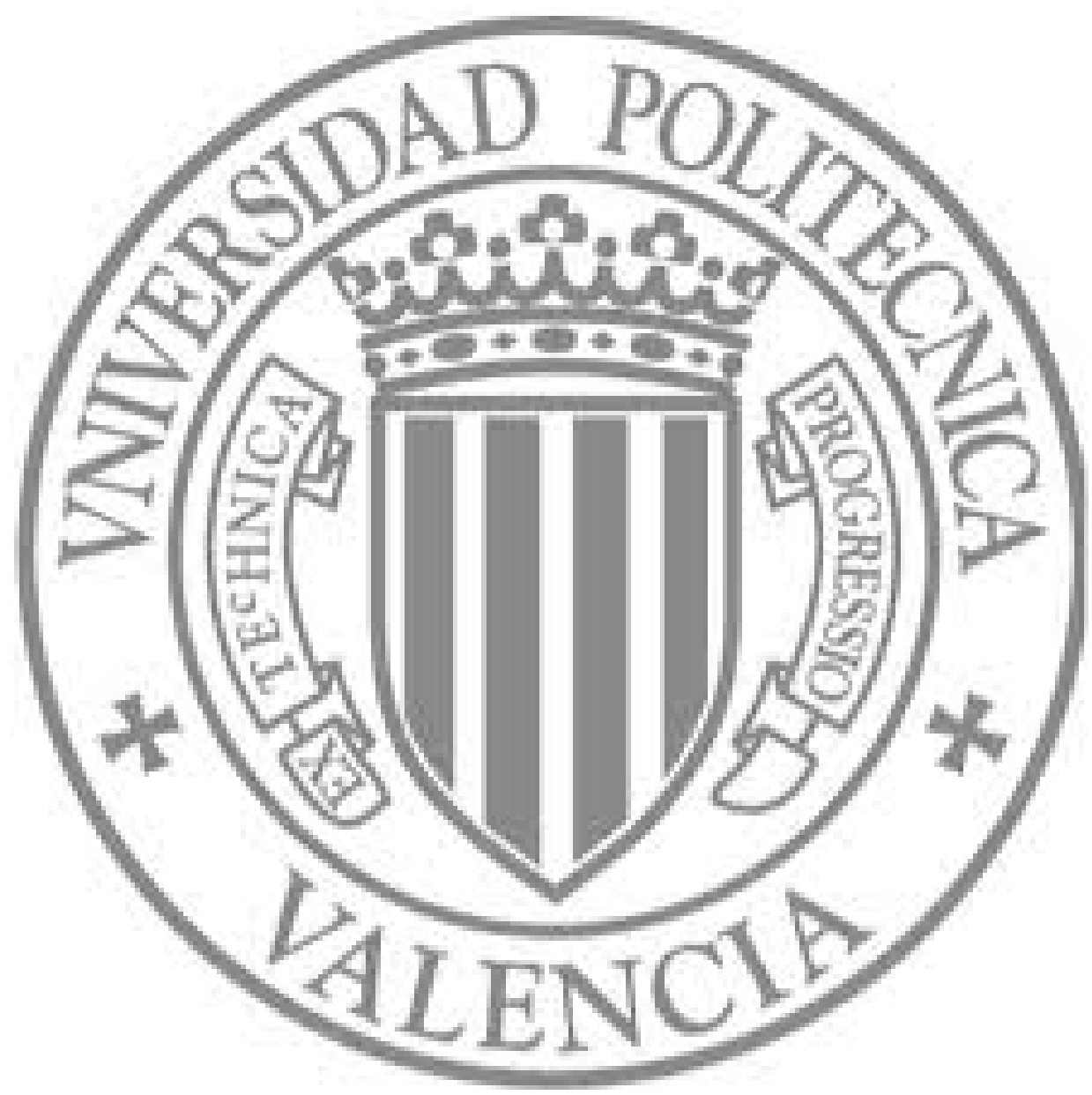

The final publication is available at

http://www.tandfonline.com/doi/abs/10.1080/01969722.2014.894859\#

Copyright Taylor \&amp;amp; Francis: STM, Behavioural Science and Public Health Titles 


\title{
An Intelligent Self-Configurable Mechanism for Distributed Energy Storage Systems
}

\author{
Juan M. Alberola, Vicente Julián, and Ana García-Fornes \\ Departament de Sistemes Informàtics i Computació, \\ Universitat Politècnica de València, \\ Camí de Vera s/n. 46022, València. Spain, \\ jalberola,vinglada, agarcia@dsic.upv.es,
}

\begin{abstract}
Next generation of smart grid technologies demand intelligent capabilities for communication, interaction, monitoring, storage, and energy transmission. Multiagent systems are envisioned to provide autonomic and adaptability features to these systems in order to gain advantage in their current environments. In this paper we present a mechanism for providing distributed energy storage systems (DESSs) with intelligent capabilities. In more detail, we propose a self-configurable mechanism which allows a DESS to adapt itself according to the future environmental requirements. This mechanism is aimed at reducing the costs at which energy is purchased from the market.
\end{abstract}

Keywords: Distributed energy storage, Multiagent Systems, Smart Grid

\section{Introduction}

Smart grid technologies are positioned as one of the leading frameworks to build the next generation of systems and applications. Intelligent functions are expected to provide the smart grid with self-corrective and reconfiguration features, 
by creating a more complex interaction behavior among intelligent devices [1]. To address these issues, the multiagent system paradigm is widely agreed to be one challenging approach to build these systems [2-6].

According to [7], one of the most important challenges for the power grid of the future is the implementation of widely distributed energy storage systems (DESSs) with intelligent monitoring, communications, and control. Storage can be applied at the energy production, at the transmission system, at the distribution system, and on the customer's side [8]. Energy storage systems in the smart grid area have been historically used with different functional and economical proposals. Among others, storage provides support to maintain and improve power quality, frequency, and voltage; storage also provides support users when failures on the network occurs, in order to improve the reliability of the power supply; or it can be used for reducing the need to buy new central generation capacity [9-11].

One of the benefits from storage that has been discussed in the literature long ago is referred to the use of storage systems for energy arbitrage. This involves purchasing electric energy during periods when the price is low, to charge the storage devices, so that the stored energy can be used or sold at a later time when the price is high [9]. However, little work has been done focused on the use of agent-based techniques for energy arbitrage modeled by DESS. To this respect, we focus on how intelligent storage systems can be build to achieve optimal configurations in the smart grid. We propose a self-configuration mechanism in order to provide a DESS with intelligent storage for improving the efficiency level. This mechanism uses an organizational representation of the DESS and focus on determining which devices are charging and supplying energy to the system at each moment. The objective of this process is aimed at scheduling the 
supplying and charging periods in order to reduce the costs for purchasing the energy demanded.

The rest of the paper is organized as follows. Section 2 analyzes some works related with our proposal. Section 3 presents the DESS model. Section 4 explains in detail the self-configuration mechanism. Section 5 shows the evaluation of the mechanism proposed. Finally, Section 6 presents some concluding remarks.

\section{Related Work}

Several works have appeared that study the benefits of DESS. In [12] authors focus on the problem of determining the scheduling of energy storage devices in order to the distribution losses are minimized. They model a DESS with distributed wind generators. Results demonstrate that distributed storage improves losses in the case of distributed generation. In [13] it is used a hybrid energy storage system, which is composed by heterogeneous storages devices, in order to reduce the cost of single storage systems. In [14] is proposed a DESS for managing large amounts of data. Other works such as [15] are focused on specification and simulation of smart grid scenarios.

Regarding arbitrage, several works have been appeared focused on this issue [16-18]. Decisions about when to store and use the energy depend on different factors such as the market prices, the storage costs, the transmission costs, etc. In addition, a storage device is typically characterized by its power capacity, its energy capacity, and its round trip efficiency among other parameters [19], which are dependent on the specific technology used for storage (pumped-hydro, compressed air, regenerative fuel cells, etc. [20,11]).

In the last few years, agent-based technologies have been used for modeling and controlling smart-grid systems. Power TAC [21] provides an environment for 
trading on energy systems. In [22] propose an agent-based simulator for smart grid environments. Most of these approaches are focused on optimizing the system performance. In [23], agents represent customers which are faced with a multi-scale decision-making problem along temporal and contextual dimensions. The objective of these agents is to maximize the utility focused on these dimensions by learning the information of time-series. In [24], authors propose a model for dynamic coalition formation to approximate optimal micro-grid configurations. In [25], an agent-based control framework is proposed to manage and coordinate the distributed energy resources.

Although the multiagent paradigm is envisioned as a strong solution for modeling DESSs because of its distribution and heterogeneity, little work has been done focused with this aim. In [26] a service restoration with distributed energy storage support is proposed. A multiagent system is proposed to detect and restore the system when a fault occurs. Ramchurn et al. [27] propose an agent-based framework in which agents are able to shift demand to times when green energy is available by using their storage devices. Results are focused on measuring green energy usage and energy cost reduction, but caused because of the use of green energy. In [28] a multiagent system for real-time control and management of a microgrid is presented. In this work, the multiagent system is proposed for demand side management and generation control. In [3], authors present an agent-based model for micro-storage management in the micro-grid. They propose a strategy based on game theory which reduces costs and carbon emission and converges to an efficient storage behavior. Their storage strategy proposed is focused on a learning mechanism that decides on when to store energy and when to use the stored energy in individual home devices. In our ap- 
proach, agents are part of a purely cooperative system, in which the best decision for the whole system does not require to affect positively to all individuals.

Our approach is focused on reducing the purchasing cost of electrical energy by coordinating and optimizing the individual devices of the DESS. Therefore, considering a predicted energy consumption and purchasing prices, the self-configuration approach must decide at each moment which devices are supplying and charging, in order to satisfy the expected demand and the system restrictions such as transferring capacity of the system.

prices and the effect that hourly charging or discharging would have on those prices. With varying prices, charges and discharges are sometimes curtailed when the price impacts reduce the marginal arbitrage value to zero. In other cases, such as on Friday morning, the device does not operate at all with varying prices, even though it would with fixed prices. [19]

\section{DESS Model}

The DESS modeled in this work represents a group of storage devices that are in charge of storing energy purchased from the market in order to supply this energy to the system (Figure 1). This system can be viewed as a whole large storage system composed by individual and heterogeneous devices. The self-configuration mechanism is aimed at deciding for any storage device, when to store energy and when to use the stored energy. The optimal configuration is dependent on the current and future energy purchasing prices, and the current and future energy demand. This represents a decision-making problem that determines the state of each storage device.

Following our previous definition of dynamic multiagent organization [29], we model the DESS as a multiagent system $G^{t}=\left\langle\mathcal{A}^{t}, \Lambda^{t}, \Delta^{t}, \Phi\right\rangle$, where: 


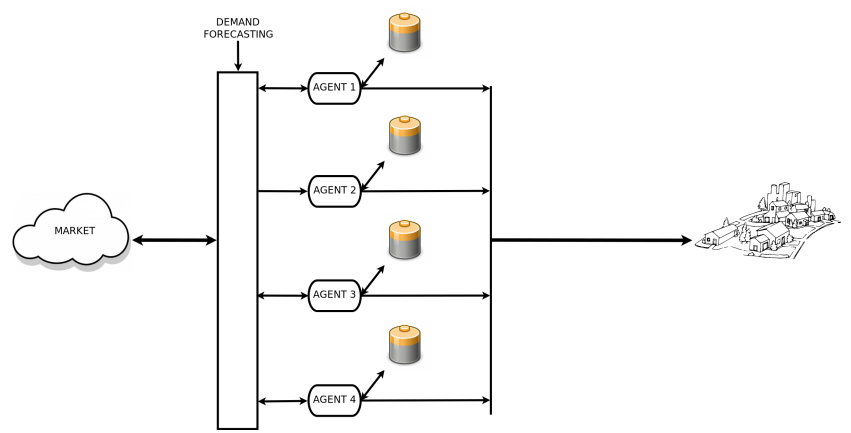

Fig. 1. Representation of the DESS.

- $\mathcal{A}^{t}=\left\{a_{1} \ldots a_{n}\right\}$ denotes the set of agents that are associated to the storage devices. Each agent $a_{x}$ is able to charge and supply energy according to the following tuple of parameters $\left\langle\right.$ max_power ${ }_{x}$, min_energy $_{x}$, max_energy $_{x}$, energy $\left.y_{x}^{t}, \operatorname{charge}_{x}^{t}, \operatorname{suppl}_{x}^{t}, p_{x}^{t}\right\rangle$, where:

- max_power ${ }_{x}$ represents the maximum power of the storage device.

- min_energy $y_{x}$ and max_energy $y_{x}$ represent the minimum energy for the storage working and the maximum energy that can be stored.

- energy $_{x}^{t}$ represents the energy available that is stored at the moment $t$.

- $\operatorname{charg} e_{x}^{t}$ represents the energy charged from the grid at the moment $t$.

- supply $y_{x}^{t}$ represents the energy supplied to the system at the moment $t$.

- $p_{x}^{t}$ represents the average price of the stored energy in $a_{x}$, according to the prices at which this energy was previously purchased:

$$
\bar{p}^{t}=\left\{\begin{array}{cc}
\lambda^{t} & \text { for } t=0 \\
\frac{\left(\text { energy } y_{x}^{t-1} \times \bar{p}^{t-1}\right)+\left(\operatorname{charge} e_{x}^{t-1} \times \lambda^{t-1}\right)-\left(\operatorname{supply}_{x}^{t-1} \times \bar{p}^{t-1}\right)}{\text { energy }_{x}^{t-1}+\operatorname{charge}_{x}^{t-1}-\operatorname{suply}_{x}^{t-1}} & \text { for } t>0
\end{array}\right.
$$

The amount of energy charged from the grid and supplied to the system determines the switch process charge/discharge. 
$-\Lambda^{t}=\lambda^{t+1} \ldots \lambda^{m}$ denotes the sequence of energy purchasing price estimations for the following moments. A given energy purchasing price $\lambda^{y}$ represents the estimated price at which the energy can be purchased from the market at the moment $y$.

$-\Delta^{t}=\delta^{t+1} \ldots \delta^{m}$ denotes the sequence of forecast demand of energy for the following moments. A given demand $\delta^{y}$ represents the forecast demand of energy at the moment $y$.

- $\Phi$ denotes the set of constraints that must be fulfilled at each moment.

\section{Self-configuration Mechanism}

The self-configuration mechanism is based on our previous work about role reallocation in agent societies [30] and is intended at providing the decision-making process that determines the state of each storage device at any moment. This mechanism provides a general vision of the whole system and allows to determine the specific consequences of each change of state in the rest of the system. It obtains the configuration of the storage devices that minimizes the energy purchasing costs, depending on the energy purchasing price and the energy demand for the forthcoming moments. The problem of predicting future energy purchasing prices is widely studied in other works such as [31-33], and is out of the scope of this work.

We define the concept of impact as a measurement of the effects of being charging or supplying energy, in terms of system utility based on the costs for carrying out each this action. This impact evaluates the different alternatives that can be chosen from the current storage devices configuration in order to adapt it, based on the benefits and costs of each alternative. 
Considering $\delta^{t+1}$ as the forecast demand of energy for the next moment $t+1$, this demand must be supplied by the energy stored in some of the storage devices: $\delta^{t+1}=\sum_{a_{x} \in A}$ supply $y_{x}^{t+1}$. The energy supplied by each agent supplier $a_{x}$ must fulfill the restrictions of the storage device. That is, this energy cannot exceed the maximum power of the storage device and the energy limitations:

$$
\operatorname{supply}_{x}^{t+1} \leq \min \left(\text { energy }_{x}^{t}-\text { min_energy }_{x}, \text { max_power }_{x}\right)
$$

In case of being a supplier, the energy stored at the agent $a_{x}$ will be reduced to: energy $_{x}^{t+1}=$ energy $y_{x}^{t}-$ supply $y_{x}^{t+1}$. This causes that each storage device needs to be charged eventually from the grid.

If the agent $a_{x}$ is charged at the moment $t+1$, the energy charged $\operatorname{charg} e_{x}^{t+1}$ must also fulfill the restrictions of the storage device:

$$
\operatorname{charg}_{x}^{t+1} \leq \min \left(\max _{-} e n e r g y_{x}-\text { energy }_{x}^{t}, \text { max_power }_{x}\right)
$$

Otherwise, if $a_{x}$ is not charged at the next moment $t+1$, the charge could be postponed to a future moment $t^{\prime}$ at which the energy purchasing price is the cheapest one. This $t^{\prime}$ is comprised in the period of time up to $t+n$, i.e. until the reserves of the DESS are running out, formally:

$$
\begin{gathered}
\left(t+2 \leq t^{\prime} \leq t+n\right) \wedge\left(\lambda_{x}^{t^{\prime}}=\underset{i \in[t+2, t+n]}{\operatorname{argmin}}\left(\lambda_{x}^{i}\right)\right) \wedge \\
\left(\sum_{i=t+2}^{i=t+n} \delta^{i} \leq \sum_{x \in A} \min \left(\text { energy }_{i}^{t}-\text { min_energy }_{x}, \text { max_power }_{x}\right)\right) \wedge \\
\left(\sum_{i=t+2}^{i=t+n+1} \delta^{i}>\sum_{x \in A} \min (\text { energy }\right.
\end{gathered}
$$

Considering the above definitions, the impact for an agent $a_{x}$ for being a supplier at the moment $t+1$ is measured as the cost required for supplying a given part of the energy demanded for the whole system:

$$
I\left(a_{x}, \text { supplier }\right)^{t+1}=\operatorname{supply}_{x}^{t+1} \times{\overline{p_{x}}}^{t} \wedge \operatorname{supply}_{x}^{t+1} \leq \delta^{t+1}
$$

In contrast, the impact for an agent $a_{x}$ for being in charge at the moment $t+1$ is measured as the cost for charging the storage device at the next moment 
subtracted from the cost for not charging the storage device in the future best moment $t^{\prime}$ :

$$
I\left(a_{x}, \operatorname{charge}\right)^{t+1}=\operatorname{charg} e_{x}^{t+1} \times \lambda^{t^{\prime}}-\operatorname{charg} e_{x}^{t+1} \times \lambda^{t+1}
$$

Given the state of the system defined as $G^{t}=\left\langle\mathcal{A}^{t}, \Lambda^{t}, \Delta^{t}, \Phi\right\rangle$, the status of each agent (supplier or charge) for the next moment $t+1$ is determined by the self-configuration mechanism. For each possible configuration $G^{t+1}$, we measure the impact of a whole self-configuration of the system as the aggregation of the impact of each allocation:

$$
I\left(G^{t+1}\right)=\sum_{a_{x} \in \mathcal{A}^{t+1}} I\left(a_{x}, \text { supplier }\right)^{t+1}+\sum_{a_{x} \in \mathcal{A}^{t+1}} I\left(a_{x}, \text { charge }\right)^{t+1}
$$

Let $\Theta$ denote the set of all the possible different configurations that can be obtained from the current state. The challenge of the self-configuration mechanism is to find the specific configuration $\widehat{G}^{t+1}$ that minimizes the whole impact:

$$
I\left(\widehat{G}^{t+1}\right)=\underset{G^{t+1} \in \Theta}{\operatorname{argmin}} I\left(G^{t+1}\right)
$$

\section{Evaluation}

In this section we present some experiments for testing the performance of the self-configuration mechanism applied to the DESS model. For these experiments, we use a system composed by a set of five agents that represent heterogeneous storage devices $\mathcal{A}^{t}=\left\{a_{0}, a_{1}, a_{2}, a_{3}, a_{4}\right\}^{1}$. The details of each storage device are given in Table 1. We assume a 100\% charging and discharging efficiency, but this could be changed by considering the corresponding energy losses. In order to simulate a realistic scenario, the system hourly load demand represents the

\footnotetext{
${ }^{1}$ Each one of these devices could be composed by a set of individual and homogeneous elements.
} 
Table 1. Configuration of the storage devices.

\begin{tabular}{|c|c|c|c|c|}
\hline Storage device & Max. Power (MW) & Max. Energy (MWh) & Min. Energy (MWh) & Initial Energy (MWh) \\
\hline$a_{0}$ & 50 & 250 & 25 & 175 \\
\hline$a_{1}$ & 100 & 500 & 50 & 250 \\
\hline$a_{2}$ & 150 & 750 & 100 & 375 \\
\hline$a_{3}$ & 200 & 1000 & 150 & 500 \\
\hline$a_{4}$ & 250 & 1250 & 200 & 625 \\
\hline
\end{tabular}

$0.1 \%$ of the real demand in the whole Spanish electric system. The purchasing price represents the hourly marginal price of the corresponding daily market auction. In this auction, a specific price is agreed for each hour of the following day. Energy storage would be economical when the marginal cost of energy varies more than the costs of storing and retrieving the energy plus the price of energy lost in the process.

For each experiment, we consider different alternatives for purchasing energy from the market. The first alternative follows a static strategy, which purchases from the market the expected demand of energy for each hour. The second alternative follows a fixed charge, which considers the self-configuration mechanism for charging the DESS depending on the expected future demand, and the expected future prices. In this alternative, a fixed transferring load is taken from the market in all hours, which corresponds to the average demand for the whole period tested. Finally, the last alternative follows a variable charge, which considers the self-configuration mechanism and considers a variable transferring load taken from the market in all hours, between 0 and 600MWh. One remarkable change in the consumers and industries demand occurs at the weekends, because most industries close, which causes a demand reduction. Therefore, in the fol- 
lowing figures we compare the demand and prices during 48 hours on weekdays and at the weekend during the month of September.

Figure 2 shows the cost of satisfying the whole demand for the three charging strategies. We can observe that the costs of the strategies that consider the selfconfiguration mechanism are almost always lower than the static charge strategy. This is because the self-configuration mechanism allows to buy and store energy when the price is expected to increase by taking into consideration the forthcoming energy demand and the purchasing price. Furthermore, the variable strategy obtains lower cost than the fixed strategy. This is because, the load taken from the market can be adjusted. In order to test this issue, Figure 3 compares the $\%$

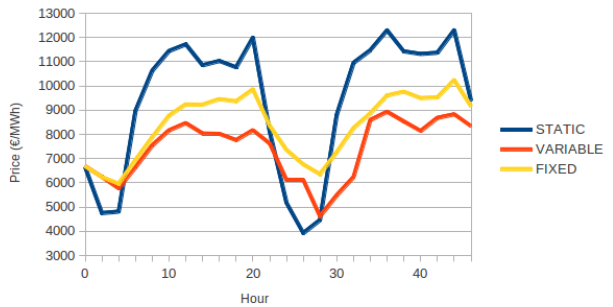

(a) Weekdays

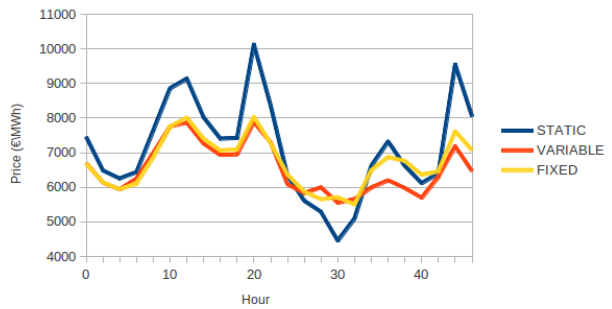

(b) Weekend

Fig. 2. Cost for satisfying the demand.

of energy (normalized between 0 and 100) that is taken from the market for the fixed and variable strategies. This figure also shows the energy price for each hour, which is normalized as well. As expected, the variable strategy takes the maximum energy for charging the DESS at those moments from which the price is going to increase in the next hours (hour 2 and 26 for the weekdays and 8, 34, and 44 at the weekend). When the price is high and there is no need to charge, 
this charge is avoided (between hour 10 and 18 for the weekdays and between hour 12 and 20 at the weekend). In contrast, the fixed strategy does not consider this deliberation because the load taken from the market is constant for each hour. This causes an increase in the aggregated cost for supplying the energy. Figure 4 shows the average price of all the energy stored in the DESS. It can

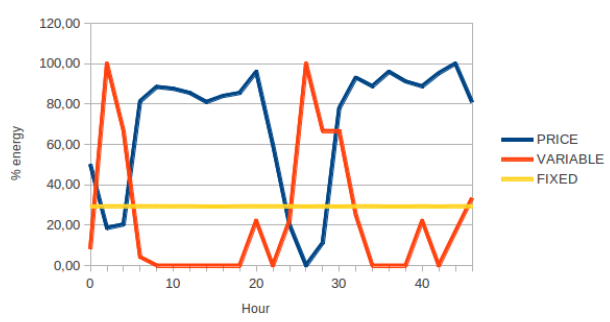

(a) Weekdays

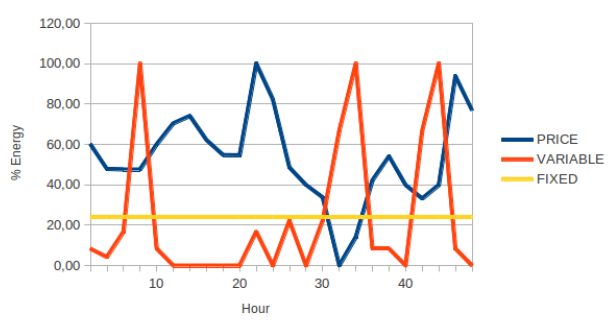

(b) Weekend

Fig. 3. Percentage of energy taken from the market.

be observed that the highest average price is obtained by the static alternative, which specially at the weekend can be around $5 € / M W h$ higher thant the alternatives that use the self-configuration mechanism. Regarding these dynamic strategies, note that although the price is quite similar for the fixed and variable strategies at the weekend, their differences are more considerable on weekdays.

The value of arbitrage for each two weekdays period period represents a saving of $4.85 € / \mathrm{MWh}$ for the fixed strategy and $9.48 € / \mathrm{MWh}$ for the variable strategy. Because of the energy demand decreases at the weekend, this saving is $2.66 € / M W h$ for the fixed strategy and 3.98€/MWh for the variable strategy.

Finally, in order to test the scalability and adaptability of the approach, we propose an scenario composed by five agents for each one of the different 


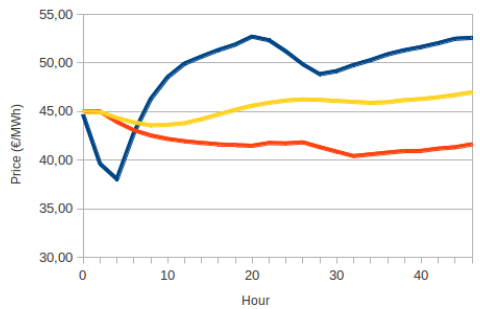

(a) Weekdays

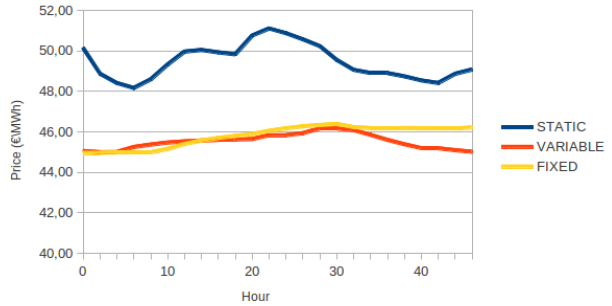

(b) Weekend

Fig. 4. Average supplying price.

storage devices, that is, 25 agents. In this scenario we test the performance of the above strategies when some agents become suddenly unavailable during 30 hours. Figure 5(a) shows the cost of satisfying the whole demand when three storage devices become unavailable at hour $=10$ and three more at hour=20. This simulation is labeled as unavailable services. We compare this simulation with a simulation without any setback during the whole execution (availability $100 \%$ ) and also with the static strategy. On the one hand, we can observe that the performance of a larger system is also better when a variable strategy is used. On the other hand, we can observe that when some setback occurs this may cause the system to reconfigure to a sub-optimal configuration. As we can observe, the cost from hour $=10$ on and from hour=20 on is a little bit higher when a setback occurs. Although this may cause a lose of efficiency, the approach is able to adapt the system in order to find a configuration that is still better than a fixed strategy.

Figure 5(b) shows the average price of all the energy stored in this scenario. Similar to the behavior of the cost, when some setback occurs, an increase in 


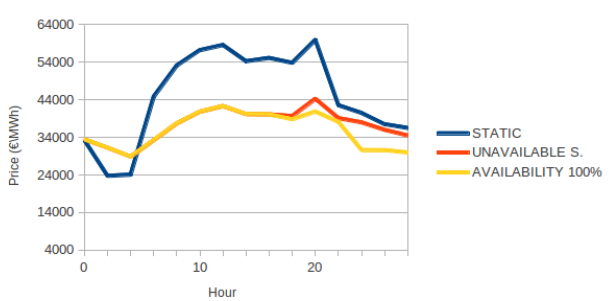

(a) Cost for satisfying the demand

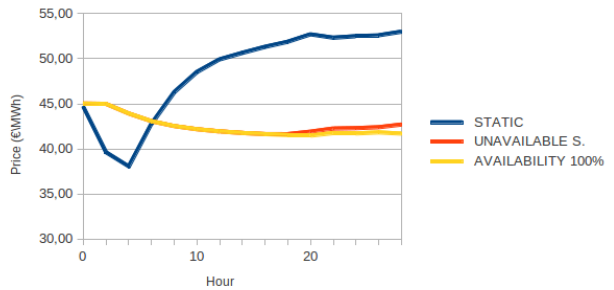

(b) Average supplying price

Fig. 5. Large-scale scenario with some services unavailable.

the price may also occur because the system may be forced to buy energy earlier than expected.

\section{Conclusions}

We proposed a self-configuration mechanism which provide distributed storage in smart grids with intelligence. The representation of the DESS by means of a multiagent organization provides different future challenges such as including other organizational dimensions to be adapted (such as the agent population) and to improve the organizational interaction and cooperation among agents.

The decision-making process associated to the self-configuration mechanism, obtains the solution which minimizes the energy supplying costs for satisfying the demand. As we observed in the experiments, these costs can be significantly reduced when taking into account the future prices and demand. In addition, since the objective is to maximize the utility of the whole system, conflicts that can emerge from individual utilities are solved due to the global view of the system. What is more, the configuration of the storage devices fits the current and future parameters of the environment by adjusting the number of devices 
that are charging and supplying energy at any moment. As a future work we plan to use this approach in a domain in which the performance of the system depends not only from the energy purchasing price but also from the energy spend, which is related with the user satisfaction criteria.

\section{Acknowledgments}

This work has been partially supported by projects TIN2012-36586-C03-01 and TIN2011-27652-C03-01.

\section{References}

[1] Momoh, J.A.: Smart grid design for efficient and flexible power networks operation and control. IEEE PES Power Systems Conference and Exposition. 15-18 (2009)

[2] Pipattanasomporn, M., Feroze, H., Rahman, S.: Multi-agent systems in a distributed smart grid: Design and implementation. IEEE/PES Power Systems Conference and Exposition. 1-8, (2009)

[3] Vytelingum, P., Voice, T.D., Ramchurn, S., Rogers, A., Jennings, N.R.: Agentbased micro-storage management for the Smart Grid. Proceedings of the 9th International Conference on Autonomous Agents and Multiagent Systems. 39-46 (2010)

[4] Vytelingum, P., Voice, T.D., Ramchurn, S., Rogers, A., Jennings, N.R.: Intelligent agents for the smart grid. Proceedings of the 9th International Conference on Autonomous Agents and Multiagent Systems. 1649-1650 (2010)

[5] Van Dam, K.H., Houwing M., Bouwmans I.: Agent-based control of distributed electricity generation with microcombined heat and power-cross-sectoral learning for process and infrastructure engineers. Computers \& Chemical Engineering. Vol. 32. 205-217, (2008)

[6] Oyarzabal, J., Jimeno, J., Ruela, J., Engler, A., Hardt, C.: Agent based Micro Grid Management System. International Conference on Future Power Systems. Vol. 18. N. 8. (2005) 
[7] Nourai, A.: Installation Of The First Distributed Energy Storage System (DESS) At American Electric Power. Technical report, Sandia National Laboratories, (2007)

[8] Mohd, A., Ortjohann, E., Schmelter, A., Hamsic, N., Morton, D.: Challenges in integrating distributed Energy storage systems into future smart grid. IEEE International Symposium on Industrial Electronics, 1627-1632, (2008)

[9] Eyer, J., Corey, G.: Energy Storage for the Electricity Grid: Benefits and Market Potential Assessment Guide. Technical report, Sandia National Laboratories, (2010)

[10] Logenthiran, T., Srinivasan, D.: Intelligent management of distributed storage elements in a smart grid. IEEE Ninth International Conference on Power Electronics and Drive Systems (PEDS). 855-860 (2011)

[11] Ribeiro, P.F., Johnson, B.K., Crow, M.L., Arsoy, A., Liu, Y.: Energy storage systems for advanced power applications. Proceedings of the IEEE. Vol. 89. N. 12 . 1744-1756 (2001)

[12] Abbey, C., Joos, G.: Coordination of distributed storage with wind energy in a rural distribution system. Industry Applications Conference, 2007. 42nd IAS Annual Meeting. Conference Record of the 2007 IEEE. 1087-1092 (2007)

[13] Vosen, S.R., Keller, J.O.: Hybrid energy storage systems for stand-alone electric power systems: optimization of system performance and cost through control strategies. International Journal of Hydrogen Energy. Vol. 24. N. 12. 1139-1156 (1999)

[14] Lakshman, A., Malik, P.: Cassandra: a decentralized structured storage system. ACM SIGOPS Operating Systems Review. Vol. 44. N. 2. 35-40 (2010)

[15] Schutte, S., Sonnenschein, M.: Mosaik-Scalable Smart Grid scenario specification. Simulation Conference (WSC), Proceedings of the 2012 Winter. 1-12 (2012)

[16] Costa, L., Bourry, F., Juban, J., Kariniotakis, G.: Management of energy storage coordinated with wind power under electricity market conditions. 10th International Conference on Probabilistic Methods Applied to Power Systems. 259-266, (2008)

[17] Pinson, P., Chevallier, C., Kariniotakis, G.N.: Trading Wind Generation From Short-Term Probabilistic Forecasts of Wind Power. IEEE Transactions on Power Systems. Vol. 22. N. 3. 1148-1156, (2007) 
[18] Maly, D.K., Kwan, K.S.: Optimal battery energy storage system (BESS) charge scheduling with dynamic programming. IEE Proceedings-Science, Measurement and Technology. Vol. 142. N. 6. 453-458 (1995)

[19] Sioshansi, R., Denholm, P., Jenkin, T., Weiss, J.: Estimating the value of electricity storage in PJM: Arbitrage and some welfare effects. Energy economics. Vol. 31. N. 2. 269-277 (2009)

[20] Chouhan, N.S., Ferdowsi, M.: Review of energy storage systems. North American Power Symposium (NAPS). 1-5 (2009)

[21] Ketter W., Collins J., Reddy, P.: Power TAC: A competitive economic simulation of the smart grid. Energy Economics. Vol. 39. N. 0. 262-270 (2013)

[22] Karnouskos, S., De Holanda, T.N.: Simulation of a smart grid city with software agents. Computer Modeling and Simulation, 2009. EMS'09. Third UKSim European Symposium on. 424-429, (2009)

[23] Reddy, P.P., Veloso. M.M.: Factored Models for Multiscale Decision Making in Smart Grid Customers. Proceedings of AAAI'12, the Twenty-Sixth AAAI Conference on Artificial Intelligence (2012)

[24] Mihailescu, R.C., Vasirani, M., Ossowski, S.: Dynamic coalition formation and adaptation for virtual power stations in smart grids. Proc. of the 2nd Int. Workshop on Agent Technologies for Energy Systems. 85-88, (2011)

[25] Jiang, Z.: Agent-based control framework for distributed energy resources microgrids. International Conference on Intelligent Agent Technology. 646-652 (2006)

[26] Nguyen, C.P., Flueck, A.J.: Agent based restoration with distributed energy storage support in smart grids. IEEE Transactions on Smart Grid. Vol. 3. N. 2. 1029-1038 (2012)

[27] Ramchurn, S.D., Vytelingum, P., Rogers, A., Jennings, N.R.: Agent-based homeostatic control for green energy in the smart grid. ACM Transactions on Intelligent Systems and Technology (TIST). Vol. 2. N. 4. 1-35 (2011)

[28] Logenthiran, T., Srinivasan, D., Khambadkone, A.M., Aung, H.N.: Agent-based homeostatic control for green energy in the smart grid. ACM Transactions on Intel- 
ligent Systems and Technology (TIST). Vol. 2. N. 4. 1-35 (2011)

[29] Alberola, J.M., Julian V., Garcia-Fornes, A.: Multi-Dimensional Adaptation in MAS Organizations. IEEE Transactions on Systems, Man, and Cybernetics, Part B: Cybernetics. In press (2013)

[30] Alberola, J.M., Julian V., Garcia-Fornes, A.: Multi-dimensional Transition Deliberation for Organization Adaptation in Multiagent Systems. Proc. 11th Int. Conf. on Aut. Agents and MAS (AAMAS12). 1379-1380, (2012)

[31] Conejo, A.J., Plazas, M.A., Espinola, R., Molina, A.B.: Day-Ahead Electricity Price Forecasting Using the Wavelet Transform and ARIMA Models. IEEE Transactions on Power Systems. Vol. 20. N. 2. 1035-1042, (2005)

[32] Mohsenian, A.H., Leon-Garcia, A.: Optimal Residential Load Control With Price Prediction in Real-Time Electricity Pricing Environments. IEEE Trans. Smart Grid. Vol. 1. N. 2. 120-133, (2010)

[33] Szkuta, B., Sanabria, L., Dillon, T.: Electricity price short-term forecasting using artificial neural networks. IEEE Transactions on Power Systems. Vol. 14. N. 3. 851857, (1999) 